\title{
The Molecular Polymorphism Evaluation in Salix Sp. Romanian Accessions - Preliminary Results
}

\author{
Mihaela CORNEANU, Sorina - Domnica POPESCU*, Cornelia HERNEA \\ Department of Genetic Engineering in Agriculture, Faculty of Horticulture and Forestry, Banat's \\ University of Agricultural Sciences and Veterinary Medicine "King Mihai I of Romania", Timisoara, \\ Romania, Calea Aradului, 119; \\ *corresponding author, e-mail: biotehnologii_usab@yahoo.com
}

Bulletin UASVM series Agriculture 73(1)/2016

Print ISSN 1843-5246; Electronic ISSN 1843-5386

DOI 10.15835/buasvmcn-agr: 11817

\begin{abstract}
Salix is an important energetic genus, but most of the genotypes are closely related genetically, due to frequent natural hybridisation in nature. Therefore it is of great interest to study its genetic background. In this work a collection of progenitors (Romanian accessions) collected from different locations were investigated to determine their genetic fingerprint in order to be used in future breeding programs. To evaluate the genetic diversity two types of molecular markers were used, namely ISSR (Inter Simple Sequence Repeats) and DAMD (Direct amplification of minisatellite-region). In the first step 15 ISSR and 5 DAMD markers were analysed to choose those markers that give the best amplification. It was pointed out that all of the primers had complex fingerprints, but those which generated the most definite patterns were A13 and UBC 818 (ISSR) and 1-URP6R and 4- 14C2 (DAMD).These primers were used to analyze seven different Salix L. samples collected from different locations. It was revealed that a high polymorphism was identified both between different accesions of a species and between species. The high degree of polymorphism for both minisatellite and microsatellite sequences, emphasized the necessity of increasing markers number to be possible to perform a statistical analysis and to determine the genetic similarities.
\end{abstract}

Keywords: Salix sp., accessions, fingerprint, molecular markers

\section{INTRODUCTION}

Due to the world energetic crisis the regenerable resources of energy, represented by vegetal biomass from SRC cultures (short rotation coppice) are a sustainable option. The species of Salix genus, constitute a promising source in the action for fighting of the environment degradation, and offer remedy for about two third from the all degradation types: erosion, destroyed as result of mining activities, industrial waste dumps, soil degradation, the presence of some ore bad smelling, sewerage works, oil exploitations, petroliferous waste dumps, waste dumps with radioactive waste, a.o. SRC willow genotypes cultivated in Romania exclusively encompasses foreign germplasm. In order to increase adaptability to a changing climate and production, as well, and to start a future breeding program, a collection of progenitors (Romanian accessions) was established.

\section{AIMS AND OBJECTIVES}

Most of the willow genotypes are closely related genetically, due to frequent natural hybridisation in nature. In order to characterize properly local germplasm the genetic evaluation based on molecular marker techniques were performed.

\section{MATERIALS AND METHODS}

The DNA was extracted from leaf tissues collected from different Salix species and locations using the CTAB method (SR EN ISO 21571). The DNA amplification followed the conditions determined by the primers specificity. In a first stage 15 ISSR and 5 DAMD markers were tested, 
choosing the primers that generated the most complex DNA fingerprints. For amplification Go Taq Green Master Mix 2x ( Promega) kit was used. The amplification conditions followed the program: $94^{\circ} \mathrm{C}-3 \mathrm{~min}, 45$ cycles: denaturation $94^{\circ} \mathrm{C}-30 \mathrm{sec}$, primers annealing $54^{\circ} \mathrm{C}-45 \mathrm{sec}$, DNA synthesis $72^{\circ} \mathrm{C}-2 \mathrm{~min}$, final synthesis $72^{\circ} \mathrm{C}$ $5 \mathrm{~min}$. The amplification products were separated by agarose gel electrophoresis and visualized with ethidium bromide.

\section{RESULTS AND DISCUSSION}

As ISSR markers we used tetra-nucleotides primers, without anchor sequence (A2 and A3), one tri-nucleotide primer, with two anchor bases at the 3' end, 10 di-nucleotides primers and two primers with degenerated sequences at the 5 " end (UBC 884 and UBC 886).

For the tetra nucleotides primers (A2 and A3) the number of generated fragments was smaller compared with the other primers. The primers with degenerated sequences (UBC 884 and UBC 886) generated complex fingerprints, with a high number of fragments of approximately similar length. Therefore their using for willow variability assessment is difficult.

For the preliminary investigations the markers were selected because they amplified a high number of fragments, with different sizes, making them suitable for variability evaluation.

As DAMD markers we used primers with sequences between 11 and 20 nucleotides, originated from the minisatellites fragments present in a high number of species. All the used primers generated fingerprints with a high number of fragments. The primer 5-M13 generated a high number of fragments, with close sizes, making difficult their evaluation. For the following screening the primers 1-URP6R (20 nucleotides) and 4-14C2 (14 nucleotides) were selected.

Further on, seven Salix L. samples were selected, collected from different locations from the West and South of Romania as follows Salix alba (Agadici), 2-Salix alba (Craiova), 3-Salix alba/ fragilis (Craiova), 4- Salix fragilis (Halda Pinoasa), 5- Salix daphnoides (Agadici), 6- Salix purpurea
(Halda Farcasesti) and 7- Salix triandra (Craiova). Two samples from Salix alba were selected because this was the most abundant specie between the collected samples. It was also chosen the sample 3 , whose phenotypic identification was not possible, probably being an interspecific hybrid.

The DNA extracted and purified from the seven samples was amplified with the 2 ISSR (A13 and UBC 818) and 2 DAMD (1-URP6R and 4-14C2) selected primers.

\section{CONCLUSION}

The fingerprints analysis pointed out a high level of polymorphism both within different accessions from a specie and between the analyzed species. The ISSR and DAMD markers could be successfully used for the molecular polymorphism evaluation of different Salix sp. accessions. The high degree of polymorphism at minisatellite sequences, as well as microsatellite sequences level, emphasized the necessity of increasing markers number and statistic analysis of obtained fragments. These will permit to determine the degree of relationships between collected genotypes.

Acknowledgements. This research was funded by the 111/2014 SAROSWE grant, by the Education and Research Ministry, UEFISCDI, Bucharest, Romania.

\section{REFERENCES}

1. Esselman EJ, Jianqiang L, Crawford DJ, Windus JL, Wolfe AD. (1999) Clonal diversity in the rare Calamagrostis porteri ssp. Insperata (Poaceae): comparative results for allozymes and random amplified polymorphic DNA (RAPD) and inter simple sequence repeat (ISSR) markers. Molecular Ecology 8: 443-451.

2. Heath DD, Iwama GK, Devlin RH (1993) PCR primed with VNTR core sequence yields species specific patterns and hypervariable probes. Nucleic Acids Res 21:5782-5785

3. ISO 21571:2005 Foodstuffs -- Methods of analysis for the detection of genetically modified organisms and derived products -- Nucleic acid extraction

4. Sulima P., J. (2013) Genetic Diversity Of Salix purpurea L. Genotypes And Interspecific Hybrids, Acta Biologica Cracoviensia, Series Botanica, 55/2: 29-36 